\title{
Variations on Classical and Quantum Extractors
}

\author{
Mario Berta \\ IQIM Caltech, Pasadena \\ Institute for Theoretical Physics, ETH Zurich
}

\author{
Omar Fawzi and Volkher B. Scholz \\ Institute for Theoretical Physics \\ ETH Zurich
}

\author{
Oleg Szehr \\ Centre for Mathematics \\ Technical University Munich
}

\begin{abstract}
Many constructions of randomness extractors are known to work in the presence of quantum side information, but there also exist extractors which do not [Gavinsky et al., STOC'07]. Here we find that spectral extractors $\psi$ with a bound on the second largest eigenvalue $\lambda_{2}\left(\psi^{\dagger} \circ \psi\right)$ are quantum-proof. We then discuss fully quantum extractors and call constructions that also work in the presence of quantum correlations decoupling. As in the classical case we show that spectral extractors are decoupling. The drawback of classical and quantum spectral extractors is that they always have a long seed, whereas there exist classical extractors with exponentially smaller seed size. For the quantum case, we show that there exists an extractor with extremely short seed size $d=O(\log (1 / \epsilon))$, where $\varepsilon>0$ denotes the quality of the randomness. In contrast to the classical case this is independent of the input size and min-entropy and matches the simple lower bound $d \geq \log (1 / \epsilon)$.
\end{abstract}

\section{INTRODUCTION}

Randomness is fundamental for many applications in computation, cryptography and information theory, and the goal of randomness extraction is to convert sources of biased and correlated bits to almost-uniform bits (see, e.g., [29]). A minentropy extractor takes an input string $N$ from a weakly random source and applies a function $f$ together with a string $D$ of perfect randomness (called seed) to yield an output string $M=f(N, D)$ which is then supposed to be $\varepsilon$-close to uniform provided that the min-entropy $H_{\min }(N)=-\log p_{\text {guess }}(N)$ is large enough. However, for some applications we also want that the extractor works if the input source is correlated to another system $R$. That is, the output should be uniform and independent of $R$ provided that the conditional min-entropy of the source $H_{\min }(N \mid R)=-\log p_{\text {guess }}(N \mid R)$ is large enough. Extractor constructions that also work if $R$ is quantum are called quantum-proof. Such extractors are crucial in classical and quantum cryptography (see, e.g., [23]) as well as for proving quantum coding theorems (see, e.g., [5], [11]). In the first part of this paper, we briefly discuss what is known about extractors (Section II) and then show that spectral extractors $\psi$ with a bound on the second largest eigenvalue $\lambda_{2}\left(\psi^{\dagger} \circ \psi\right)$ are quantum proof (Section III). In the second part of this paper (Sections IV and $\mathrm{V}$, we consider fully quantum minentropy extractors that output a quantum state that is $\varepsilon$-close to maximally mixed from a quantum source $\rho_{N}$ provided that the min-entropy $H_{\min }(N)_{\rho}=-\log \lambda_{1}\left(\rho_{N}\right)$ is large enough $\left(\lambda_{1}(\cdot)\right.$ denotes the largest eigenvalue). We then discuss an extension of this when the input source is correlated to another quantum system $R$, and we require the output to be to uniform and independent of $R$ provided that the quantum conditional min-entropy $H_{\min }(N \mid R)_{\rho}=-\log F(N, R)_{\rho}$ is large enough $\left(F(N, R)_{\rho}\right.$ denotes the maximal achievable singlet fraction [18]). We call such extractors decoupling and show that spectral extractors are decoupling. Finally, we show that spectral extractors have the drawback of a long seed, but also give a direct extractor construction with extremely short seed. We end by stating some open questions (Section VI).

We use the following notation. The labels $N, M, D$ are used to specify the subsystem as well as the domain of the classical system, and classical states on $N$, i.e., probability distributions on $N$, are denoted by $P_{N} \in \ell^{1}(N)$. The normalized uniform distribution on $N$ is denoted by $u_{N}$, and the set of distributions on $N$ is denoted by $\ell(N)$. Quantum systems are represented by their (finite-dimensional) Hilbert spaces $\mathcal{H}_{N}, \mathcal{H}_{M}, \mathcal{H}_{R}$, and states on $\mathcal{H}_{N}$, i.e., non-negative trace-one operators on $\mathcal{H}_{N}$ are denoted by $\rho_{N} \in \mathcal{S}\left(\mathcal{H}_{N}\right)$. We denote the set of linear operators on $\mathcal{H}_{N}$ by $\mathcal{P}\left(\mathcal{H}_{N}\right)$. For $\alpha \geq 1$ and $\sigma \in \mathcal{P}(\mathcal{H})$ with $\sigma \geq 0$ we have the $\sigma$-weighted $\alpha$-norms

$$
\|\cdot\|_{\alpha, \sigma}=\left(\operatorname{tr}\left[\left|\sigma^{1 / 2 \alpha}(\cdot) \sigma^{1 / 2 \alpha}\right|^{\alpha}\right]\right)^{1 / \alpha},
$$

and for $\alpha=2$ the norm is induced by the $\sigma$-weighted HilbertSchmidt inner product

$$
\langle\cdot \mid *\rangle_{\sigma}=\operatorname{tr}\left[\left(\sigma^{1 / 4}(\cdot) \sigma^{1 / 4}\right)^{\dagger}\left(\sigma^{1 / 4}(*) \sigma^{1 / 4}\right)\right] .
$$

The $\sigma$-weighted $\alpha$-norms satisfy the Hölder inequalities

$$
\langle\cdot \mid *\rangle_{\sigma} \leq\|\cdot\|_{p, \sigma} \cdot\|*\|_{q, \sigma}
$$

for $1 / p+1 / q=1$ [21]. For $\rho_{N R} \in \mathcal{S}\left(\mathcal{H}_{N R}\right)$ the quantum conditional min-entropy is defined as [18]

$$
H_{\min }(N \mid R)_{\rho}=-\log \max _{\substack{E_{N R} \geq 0 \\ \operatorname{tr}_{N}\left[E_{N R}\right]=\mathbb{1}_{R}}} \operatorname{tr}\left[\rho_{N R} E_{N R}\right]
$$

and the classical-quantum version simplifies to $H_{\text {min }}(N \mid R)_{\rho}=-\log p_{\text {guess }}(N \mid R)_{\rho}$, where $p_{\text {guess }}(N \mid R)_{\rho}$ is the maximal probability of decoding $N$ from measurements on $R$. For $\rho_{N R} \in \mathcal{S}\left(\mathcal{H}_{N R}\right)$ the quantum conditional Rényitwo entropy is defined as $H_{2}(N \mid R)_{\rho}=-\log \left\|\tilde{\rho}_{N R}\right\|_{2}^{2}$ with $\tilde{\rho}_{N R}=\left(\mathbb{1}_{N} \otimes \rho_{R}^{-1 / 4}\right) \rho_{N R}\left(\mathbb{1}_{N} \otimes \rho_{R}^{-1 / 4}\right)$, and we have [4]

$$
H_{\min }(N \mid R)_{\rho} \leq H_{2}(N \mid R)_{\rho} \text {. }
$$

\section{Classical Min-Entropy Extractors}

The definition of a (strong) extractor is due to Nisan and Zuckerman [20]. 
Definition 1. Let $M \subset N, k \in[0, \log |N|]$, and $\varepsilon>0$. A $(k, \varepsilon)$ extractor is a set of functions $\left\{f_{1}, \ldots, f_{|D|}\right\}$ from $N$ to $M$ such that for all $P_{N} \in \ell^{1}(N)$ with $H_{\min }(N)_{P} \geq k$,

$$
\| \frac{1}{|D|} \cdot \sum_{i=1}^{|D|} P_{f_{i}(N)} \otimes|i\rangle\left\langle\left. i\right|_{D}-u_{M} \otimes u_{D} \|_{1} \leq \varepsilon .\right.
$$

The quantity $n=\log |N|$ is called the input size, $m=\log |M|$ the output size, and $d=\log |D|$ the seed size ${ }^{1}$

An extractor is called permutation based if all the functions $f_{i}: N \rightarrow M$ have the form $f_{i}(\cdot)=\left.\pi_{i}(\cdot)\right|_{M}$ with $\pi_{i} \in S_{|N|}$, the symmetric group on $\{1,2, \ldots,|N|\}$. It is instructive to consider extractors with domain and range consisting of bit strings, that is, $N=\{0,1\}^{n}, M=\{0,1\}^{m}, D=\{0,1\}^{d}$. Typically we are given fixed $n, k$, and $\varepsilon$, and we want to maximize the output length $m$ and minimize the seed length $d$. Radhakrishnan and Ta-Shma gave an ultimate limit on $m$ and $d$ : every $(k, \varepsilon)$ extractor necessarily has

$$
\begin{aligned}
& m \leq k-2 \log \frac{1}{\varepsilon}+O(1) \\
& d \geq \log (n-k)+2 \log \frac{1}{\varepsilon}-O(1) .
\end{aligned}
$$

It turns out that a probabilistic construction using random functions achieves these bounds up to constants: there exists a $(k, \varepsilon)$ extractor with [22], [24]

$$
\begin{aligned}
& m=k-2 \log \frac{1}{\varepsilon}-O(1) \\
& d=\log (n-k)+2 \log \frac{1}{\varepsilon}+O(1) .
\end{aligned}
$$

However, for applications we usually want explicit extractors and starting with Trevisan's breakthrough result [28] there are now many constructions that almost achieve the bounds above (see [29] and references therein). Here, we study the question if extractors also work in the presence of quantum information.

\section{QuANTUM-Proof EXTRactors}

Definition 2. A $(k, \varepsilon)$ extractor $\left\{f_{1}, \ldots, f_{|D|}\right\}$ is quantumproof if for all classical-quantum states $\rho_{N R} \in \mathcal{S}\left(\mathcal{H}_{N R}\right)$ with $H_{\min }(N \mid R)_{\rho} \geq k$,

$$
\| \frac{1}{|D|} \cdot \sum_{i=1}^{|D|} \rho_{f_{i}(N) R} \otimes|i\rangle\left\langle\left. i\right|_{D}-u_{M} \otimes \rho_{R} \otimes u_{D} \|_{1} \leq \varepsilon .\right.
$$

It was shown by König and Terhal that extractors with one bit output are quantum-proof [19, Theorem 1], and in general it is known by now that many extractor constructions are quantum-proof [17], [19], [23], [25], [27] or suffer at most from a decent parameter loss [7]. However, Gavinsky et al. [13] gave an example of a valid (though contrived) extractor that completely fails in the presence of quantum side information. Moreover there is no general understanding of

\footnotetext{
${ }^{1}$ For a weak $(k, \varepsilon)$ extractor [6 is replaced with $\| \frac{1}{|D|} \cdot \sum_{i=1}^{|D|} P_{f_{i}(N)}-$ $u_{M} \|_{1} \leq \varepsilon$ (i.e., the seed system $D$ is not necessarily part of the output).
}

when an extractor is quantum-proof. Next, we define spectral extractors and show that they are quantum-proof extractors.

Definition 3. A $(k, \varepsilon)$ spectral extractor is a set of functions $\left\{f_{1}, \ldots, f_{|D|}\right\}$ from $N$ to $M$ such that for the map $\psi$ : $\ell(N) \rightarrow \ell(M D)$ with $\psi\left(P_{N}\right)=\frac{1}{|D|} \cdot \sum_{i=1}^{|D|} P_{f_{i}(N)} \otimes|i\rangle\left\langle\left. i\right|_{D}\right.$,

$$
\lambda_{1}\left(\psi^{\dagger} \circ \psi-\tau^{\dagger} \circ \tau\right) \leq 2^{k} \cdot \frac{\varepsilon}{|M| \cdot|D|},
$$

where $\lambda_{1}(\cdot)$ denotes the largest eigenvalue, and $\tau\left(P_{N}\right)=$ $\left(\sum_{j=1}^{|N|} P_{j}\right) \cdot\left(u_{M} \otimes u_{D}\right)$. For typical applications, it is sufficient to bound the second largest eigenvalue $\lambda_{2}\left(\psi^{\dagger} \circ \psi\right)$.

Now, we show that spectral extractors are also quantumproof extractors. For this we use a similar calculation as Renner et al., who showed (directly) that families of twouniversal hash functions [23], [27], and families of pairwise independent permutations [25] give rise to quantum-proof extractors.

Theorem 4. Every $(k, \varepsilon)$ spectral extractor is also a quantumproof $(k, 2 \sqrt{\varepsilon})$ extractor of the same output size and the same seed size.

Proof: We write the extractor as a map $\psi: \ell(N) \rightarrow$ $\ell(M D)$ with $\psi\left(P_{N}\right)=\frac{1}{|D|} \cdot \sum_{i=1}^{|D|} P_{f_{i}(N)} \otimes|i\rangle\left\langle\left. i\right|_{D}\right.$, and denote $\tau: \ell(N) \rightarrow \ell(M D)$ with $\tau\left(P_{N}\right)=\left(\sum_{j=1}^{|N|} P_{j}\right) \cdot\left(u_{M} \otimes u_{D}\right)$. Then we get for the 1-norm

$$
\begin{aligned}
& \left\|\left((\psi-\tau) \otimes \mathcal{I}_{R}\right)\left(\rho_{N R}\right)\right\|_{1} \\
& =2 \cdot \max _{0 \leq X \leq \mathbb{1}} \operatorname{tr}\left[\left((\psi-\tau) \otimes \mathcal{I}_{R}\right)\left(\rho_{N R}\right) X\right] \\
& =2 \cdot \max _{0 \leq X \leq \mathbb{1}} \operatorname{tr}\left[\left((\psi-\tau) \otimes \mathcal{I}_{R}\right)\left(\hat{\rho}_{N R}\right)\left(\mathbb{1}_{M D} \otimes \rho_{R}^{1 / 2}\right)\right. \\
& \left.\quad X\left(\mathbb{1}_{M D} \otimes \rho_{R}^{1 / 2}\right)\right] \\
& =2 \cdot \max _{0 \leq X \leq \mathbb{1}}\left\langle\left((\psi-\tau) \otimes \mathcal{I}_{R}\right)\left(\hat{\rho}_{N R}\right) \mid X\right\rangle_{(\mathbb{1} \otimes \rho)},
\end{aligned}
$$

where $\hat{\rho}_{N R}=\left(\mathbb{1}_{N} \otimes \rho_{R}^{-1 / 2}\right) \rho_{N R}\left(\mathbb{1}_{N} \otimes \rho_{R}^{-1 / 2}\right)$, and we made use of the $\left(\mathbb{1}_{M D} \otimes \rho_{R}\right)$-weighted Hilbert-Schmidt inner product. By using the $(2,2)$-Hölder inequality for the $\left(\mathbb{1}_{M D} \otimes \rho_{R}\right)$ weighted Hilbert-Schmidt inner product we get

$$
\begin{aligned}
& 2 \cdot \max _{0 \leq X \leq \mathbb{1}}\left\langle\left((\psi-\tau) \otimes \mathcal{I}_{R}\right)\left(\hat{\rho}_{N R}\right) \mid X\right\rangle_{(\mathbb{1} \otimes \rho)} \\
& \leq 2 \cdot \max _{0 \leq X \leq \mathbb{1}}\|X\|_{2,(\mathbb{1} \otimes \rho)} \cdot\left\|\left((\psi-\tau) \otimes \mathcal{I}_{R}\right)\left(\hat{\rho}_{N R}\right)\right\|_{2,(\mathbb{1} \otimes \rho)},
\end{aligned}
$$

with the $\left(\mathbb{1}_{M D} \otimes \rho_{R}\right)$-weighted 2-norm. We estimate the first term by using the $(1, \infty)$-Hölder inequality for the $\left(\mathbb{1}_{M D} \otimes\right.$ $\rho_{R}$ )-weighted Hilbert-Schmidt inner product

$$
\begin{aligned}
\|X\|_{2,(\mathbb{1} \otimes \rho)} & =\sqrt{\langle X \mid X\rangle_{2,(\mathbb{1} \otimes \rho)}} \\
& \leq \sqrt{\|X\|_{\infty,(\mathbb{1} \otimes \rho)} \cdot\|X\|_{1,(\mathbb{1} \otimes \rho)}} \\
& =\sqrt{\lambda_{1}(X) \cdot \operatorname{tr}\left[\left(\mathbb{1}_{M D} \otimes \rho_{R}\right) X\right]} \\
& \leq \sqrt{|M| \cdot|D|} .
\end{aligned}
$$


For the second term a straightforward calculation gives

$$
\begin{aligned}
\| & \left((\psi-\tau) \otimes \mathcal{I}_{R}\right)\left(\hat{\rho}_{N R}\right) \|_{2,(\mathbb{1} \otimes \rho)}^{2} \\
= & \left\langle\left(\psi \otimes \mathcal{I}_{R}\right)\left(\hat{\rho}_{N R}\right) \mid\left(\psi \otimes \mathcal{I}_{R}\right)\left(\hat{\rho}_{N R}\right)\right\rangle_{(\mathbb{1} \otimes \rho)} \\
& -2 \cdot\left\langle\left(\psi \otimes \mathcal{I}_{R}\right)\left(\hat{\rho}_{N R}\right) \mid\left(\tau \otimes \mathcal{I}_{R}\right)\left(\hat{\rho}_{N R}\right)\right\rangle_{(\mathbb{1} \otimes \rho)} \\
& +\left\langle\left(\tau \otimes \mathcal{I}_{R}\right)\left(\hat{\rho}_{N R}\right) \mid\left(\tau \otimes \mathcal{I}_{R}\right)\left(\hat{\rho}_{N R}\right)\right\rangle_{(\mathbb{1} \otimes \rho)} \\
= & \left\langle\hat{\rho}_{N R} \mid\left(\left(\psi^{\dagger} \circ \psi-\tau^{\dagger} \circ \tau\right) \otimes \mathcal{I}_{R}\right)\left(\hat{\rho}_{N R}\right)\right\rangle_{(\mathbb{1} \otimes \rho)} \\
= & \left\langle\tilde{\rho}_{N R} \mid\left(\left(\psi^{\dagger} \circ \psi-\tau^{\dagger} \circ \tau\right) \otimes \mathcal{I}_{R}\right)\left(\tilde{\rho}_{N R}\right)\right\rangle,
\end{aligned}
$$

where $\tilde{\rho}_{N R}=\left(\mathbb{1}_{N} \otimes \rho_{R}^{-1 / 4}\right) \rho_{N R}\left(\mathbb{1}_{N} \otimes \rho_{R}^{-1 / 4}\right)$. By (5) the conditional min-entropy is upper bounded by the conditional Rényi-two entropy $H_{2}(N \mid R)_{\rho}=-\log \left\|\tilde{\rho}_{N R}\right\|_{2}^{2}$, and this gives

$$
\begin{aligned}
& \sup _{\substack{H_{\min }(N \mid R)_{\rho} \geq k \\
\|\rho\|_{1}=1}}\left\langle\tilde{\rho}_{N R} \mid\left(\left(\psi^{\dagger} \circ \psi-\tau^{\dagger} \circ \tau\right) \otimes \mathcal{I}_{R}\right)\left(\tilde{\rho}_{N R}\right)\right\rangle \\
& \leq \frac{1}{2^{k}} \cdot \sup _{\|\tilde{\rho}\|_{2}=1}\left\langle\tilde{\rho}_{N R} \mid\left(\left(\psi^{\dagger} \circ \psi-\tau^{\dagger} \circ \tau\right) \otimes \mathcal{I}_{R}\right)\left(\tilde{\rho}_{N R}\right)\right\rangle \\
& =\frac{1}{2^{k}} \cdot \lambda_{1}\left(\psi^{\dagger} \circ \psi-\tau^{\dagger} \circ \tau\right) .
\end{aligned}
$$

From the properties of a $(k, \varepsilon)$ spectral extractor, the claim follows.

An instructive example are two-universal families of hash functions.

Definition 5. A set of functions $\left\{f_{1}, \ldots, f_{|D|}\right\}$ from $N$ to $M$ is said to be a two-universal family of hash functions if we have for all $j \neq k \in N$,

$$
\frac{1}{D} \sum_{i=1}^{|D|} \delta_{f_{i}(j)=f_{i}(k)} \leq \frac{1}{|M|} .
$$

Proposition 6. A two-universal family of hash functions is a $(k, \varepsilon)$ spectral extractor with $m=k-\log (1 / \varepsilon)$.

Proof: For any $P_{N}, Q_{N} \in \ell(N)$ we calculate

$$
\left\langle Q_{N} \mid\left(\tau^{\dagger} \circ \tau\right)\left(P_{N}\right)\right\rangle=\frac{1}{|M| \cdot|D|} \cdot \sum_{j=1}^{|N|} P_{N}^{j} \sum_{k=1}^{|N|} Q_{N}^{k} .
$$

Furthermore, we get from [18),

$$
\begin{aligned}
& \left\langle Q_{N} \mid\left(\psi^{\dagger} \circ \psi\right)\left(P_{N}\right)\right\rangle=\frac{1}{|D|^{2}} \cdot \sum_{i=1}^{|D|}\left\langle P_{N} \mid\left(\psi_{i}^{\dagger} \circ \psi_{i}\right)\left(Q_{N}\right)\right\rangle \\
& =\frac{1}{|D|^{2}} \cdot \sum_{i=1}^{|D|} \sum_{j, k=1}^{|N|} \bar{P}_{N}^{j} Q_{N}^{k} \delta_{f_{i}}(j)=f_{i}(k) \\
& \leq \frac{1}{|D|} \cdot\left\langle P_{N} \mid Q_{N}\right\rangle+\frac{1}{|M| \cdot|D|} \cdot \sum_{j=1}^{|N|} P_{N}^{j} \sum_{k=1}^{|N|} Q_{N}^{k}
\end{aligned}
$$

Hence, we arrive at

$$
\left\langle P_{N} \mid\left(\psi^{\dagger} \circ \psi-\tau^{\dagger} \circ \tau\right)\left(P_{N}\right)\right\rangle \leq \frac{1}{|D|} \cdot\left\langle X_{N} \mid X_{N}\right\rangle,
$$

and the claim follows.

Other examples of constructions based on spectral extractors are, e.g., pairwise independent families of permutations, or constructions based on balanced expander graphs (these are weak extractors). Unfortunately, spectral extractors have the drawback of a long seed.

Proposition 7. Every $(k, \varepsilon)$ spectral extractor with input size $n$, output size $m$, and seed size $d$ necessarily has

$$
d \geq \min \{n-k, m\}+\log \frac{1}{\varepsilon}-O(1) .
$$

Proof: By the same arguments as in the proof of Theorem 4 we have that

$$
\lambda_{1}\left(\psi^{\dagger} \circ \psi-\tau^{\dagger} \circ \tau\right)=\sup _{\left\|P_{N}\right\|_{2}^{2} \leq 1}\left\|(\psi-\tau)\left(P_{N}\right)\right\|_{2}^{2},
$$

where $P_{N} \in \ell(N)$. Now let $Q_{N}$ be a flat $k$-source (i.e., $Q_{N}$ has $2^{k}$ non-zero entries equal to $2^{-k}$ ) such that the image $Q_{f_{1}(N)}$ of $Q_{N}$ under the function $f_{1}$ has support of size $|S|=$ $\left\lceil 2^{k} \cdot|M| /|N|\right\rceil$. Since $H_{2}(N)_{Q}=-\log \left\|Q_{N}\right\|_{2}^{2} \geq k$ we have with (23) that

$$
\begin{aligned}
\lambda_{1}\left(\psi^{\dagger} \circ \psi-\tau^{\dagger} \circ \tau\right) & \geq \frac{2^{k}}{|D|^{2}} \cdot \sum_{i=1}^{|D|}\left\|Q_{f_{i}(N)}-u_{M}\right\|_{2}^{2} \\
& \geq \frac{2^{k}}{|D|^{2}} \cdot\left\|Q_{f_{1}(N)}-u_{M}\right\|_{2}^{2} \\
& \geq \frac{2^{k}}{|D|^{2}} \cdot \sum_{s=1}^{|S|}\left(Q_{f_{1}(N)}^{s}-\frac{1}{|M|}\right)^{2} \\
& \geq \frac{2^{k}}{|D|^{2}} \cdot|S| \cdot\left(\frac{1}{|S|}-\frac{1}{|M|}\right)^{2},
\end{aligned}
$$

where we used for the last inequality that the expression is maximized when $Q_{f_{1}(N)}$ is uniformly distributed over $S$. Hence, we get by assumption that

$$
\frac{1}{|D|} \cdot|S| \cdot\left(\frac{1}{|S|}-\frac{1}{|M|}\right)^{2} \leq \frac{\varepsilon}{|M|}
$$

or equivalently,

$$
|D| \geq \frac{1}{\varepsilon} \cdot \frac{(|M|-|S|)^{2}}{|M| \cdot|S|}
$$

Now, if $2^{k} \cdot|M| /|N| \leq 1$, then $|S|=1$ and (26) becomes

$$
\begin{aligned}
d & \geq \log \frac{1}{\varepsilon}+2 \log (|M|-1)-\log |M| \\
& \geq m+\log \frac{1}{\varepsilon}-2 .
\end{aligned}
$$

Otherwise, we get

$$
d \geq \log \frac{1}{\varepsilon}+\log \left(\frac{N}{2^{k}} \cdot \frac{\left(1-2^{k} /|N|-1 /|M|\right)^{2}}{1+|N| /\left(2^{k} \cdot|M|\right)}\right) .
$$

But we have $\frac{|N|}{2^{k} \cdot|M|} \leq 1$ and thus,

$$
\frac{\left(1-2^{k} /|N|-1 /|M|\right)^{2}}{1+|N| /\left(2^{k} \cdot|M|\right)} \geq \frac{1-2 \cdot 2^{k} / N}{2} .
$$

When $k \leq n-2$, then we get

$$
d \geq \log \frac{1}{\varepsilon}+\log \left(\frac{N}{2^{k}} \cdot \frac{1}{4}\right)=n-k+\log \frac{1}{\varepsilon}-2,
$$

and otherwise the bound we aim to prove is simply implied by the general lower bound for the seed of extractors 7 . 


\section{QuANTUM Min-EnTROPY EXTRACTORS}

To understand our definition of quantum extractors, it is convenient to start with permutation based classical extractors, i.e., a family of permutations acting on the input. This family of permutations should satisfy the following property: for any probability distribution on input bit strings with high minentropy, applying a typical permutation from the family to the input induces an almost uniform probability distribution on a prefix of the output. We define a quantum to quantum extractor in a similar way by allowing the operations performed to be general unitary transformations and the input to the extractor to be quantum.

Definition 8. Let $\mathcal{H}_{M} \subset \mathcal{H}_{N}, k \in[0, \log |N|]$, and $\varepsilon>0$. A $(k, \varepsilon)$-quantum extractor is a set of unitaries $\left\{U_{N}^{1}, \ldots, U_{N}^{|D|}\right\}$ such that for all $\rho_{N} \in \mathcal{S}\left(\mathcal{H}_{N}\right)$ with $H_{\min }(N)_{\rho} \geq k$,

$$
\| \frac{1}{|D|} \cdot \sum_{i=1}^{|D|} \operatorname{tr}_{N \backslash M}\left[U_{N}^{i} \rho_{N}\left(U_{N}^{i}\right)^{\dagger}\right] \otimes|i\rangle\left\langle\left. i\right|_{D}-\frac{\mathbb{1}_{M}}{|M|} \otimes \frac{\mathbb{1}_{D}}{|D|} \|_{1}\right.
$$$$
\leq \varepsilon \text {. }
$$

The quantity $n=\log |N|$ is called the input size, $m=\log |M|$ the output size, and $d=\log |D|$ the seed size 2

We note that the seed $D$ is still classical in this definition. Alternatively, we could also define quantum extractors as general quantum channels from $\mathcal{S}\left(\mathcal{H}_{N}\right)$ to $\mathcal{S}\left(\mathcal{H}_{M}\right)$, and the number of Kraus operators would correspond to the dimension of the quantum seed $D$. For example, the fully depolarizing channel corresponds to a perfect extractor, independent of the min-entropy of the input. But since the minimal number of Kraus operators of the fully depolarizing channel is equal to the square of the output dimension $|M|$, it also has quantum seed size $d=2 \mathrm{~m}$. However, here we restrict ourselves to quantum extractors with classical seed. It is instructive to consider extractors with domain and range consisting of qubit strings, i.e., $\mathcal{H}_{N}=\left(\mathbb{C}^{2}\right)^{\otimes n}$ and $\mathcal{H}_{M}=\left(\mathbb{C}^{2}\right)^{\otimes m}$, as well as with a binary seed, i.e., $D=\{0,1\}^{d}$. Examples for quantum extractors in the literature include the following:

- In [10] so-called decoupling theorems were studied, and in particular it was shown that unitary 2-designs (see Definition 12) are quantum extractors.

- Ben-Aroya et al. considered weak quantum extractors with the input size equal to the output size [3, Definition 5.1], and showed how to use quantum expanders for explicit constructions. See also the related work by Harrow [15] and references therein.

- Hayden et al. studied quantum state randomization, which corresponds to weak $(0, \varepsilon)$-quantum extractors with the input size equal to the output size [16]. See also the subsequent literature [1], [2], [8].

\footnotetext{
${ }^{2}$ For a weak $(k, \varepsilon)$-quantum extractor we just replace 31 with $\| \frac{1}{|D|}$ $\sum_{i=1}^{|D|} \operatorname{tr}_{N \backslash M}\left[U_{N}^{i} \rho_{N}\left(U_{N}^{i}\right)^{\dagger}\right]-\frac{\mathbb{1}_{M}}{|M|} \|_{1} \leq \varepsilon$.
}

These constructions have many applications in quantum information theory, quantum cryptography, quantum complexity theory, and quantum physics (see, e.g., the papers above and references therein). Here, we discuss whether an extractor also works if the input is initially correlated with another quantum system. That is, we ask if an extractor is not only randomizing but decoupling as well. Note that the fully quantum conditional min-entropy can be negative for entangled states.

Definition 9. Let $\mathcal{H}_{M} \subset \mathcal{H}_{N}, k \in[-\log |N|, \log |N|]$, and $\varepsilon>0$. A $(k, \varepsilon)$-quantum extractor $\left\{U_{N}^{1}, \ldots, U_{N}^{|D|}\right\}$ is decoupling if for all $\rho_{N R} \in \mathcal{S}\left(\mathcal{H}_{N R}\right)$ with $H_{\min }(N \mid R)_{\rho} \geq k$,

$$
\begin{aligned}
\| \frac{1}{|D|} \cdot \sum_{i=1}^{|D|} \operatorname{tr}_{N \backslash M}\left[U_{N}^{i} \rho_{N R}\left(U_{N}^{i}\right)^{\dagger}\right] \otimes|i\rangle\left\langle\left. i\right|_{D}\right. \\
-\frac{\mathbb{1}_{M}}{|M|} \otimes \rho_{R} \otimes \frac{\mathbb{1}_{D}}{|D|} \|_{1} \leq \varepsilon .
\end{aligned}
$$

Decoupling quantum extractors are extremely useful in quantum coding theory (see, e.g., [9] and references therein). In analogy to the classical case, one way of constructing quantum extractors is by means of quantum spectral extractors. In fact, all constructions (even the probabilistic ones) for quantum extractors that are known to be decoupling are based on spectral extractors.

Definition 10. A $(k, \varepsilon)$-quantum spectral extractor is a set of unitaries $\left\{U_{N}^{1}, \ldots, U_{N}^{|D|}\right\}$ such that for the map $\psi\left(\rho_{N}\right)=$ $\frac{1}{|D|} \cdot \sum_{i=1}^{|D|} \operatorname{tr}_{N M}\left[U_{N}^{i} \rho_{N}\left(U_{N}^{i}\right)^{\dagger}\right] \otimes|i\rangle\left\langle\left. i\right|_{D}\right.$,

$$
\lambda_{1}\left(\psi^{\dagger} \circ \psi-\tau^{\dagger} \circ \tau\right) \leq 2^{k} \cdot \frac{\varepsilon}{|M| \cdot|D|},
$$

where $\tau\left(\rho_{N}\right)=\operatorname{tr}\left[\rho_{N}\right] \cdot \frac{\mathbb{1}_{M}}{|M|} \otimes \frac{\mathbb{1}_{D}}{|D|}$. For typical applications, it is sufficient to bound the second largest eigenvalue $\lambda_{2}\left(\psi^{\dagger} \circ \psi\right)$.

In full analogy to the classical case, we get:

Theorem 11. Every $(k, \varepsilon)$-quantum spectral extractor is also a decoupling $(k, 2 \sqrt{\varepsilon})$-quantum extractor of the same output size and the same seed size.

An instructive example are unitary two-designs.

Definition 12. A set of unitaries $\left\{U_{1}, \ldots, U_{L}\right\}$ acting on $\mathcal{H}$ is said to be a unitary 2-design if we have for all $M \in \mathcal{B}(\mathcal{H})$ that

$$
\frac{1}{L} \cdot \sum_{i=1}^{L} U_{i}^{\otimes 2} M\left(U_{i}^{\dagger}\right)^{\otimes 2}=\int U^{\otimes 2} M\left(U^{\dagger}\right)^{\otimes 2} d U,
$$

where the integration is with respect to the Haar measure on the unitary group.

Many efficient constructions of unitary 2-designs are known [6], [14], and in an $n$-qubit space, such unitaries can typically be computed by circuits of size $O\left(n^{2}\right)$.

Proposition 13. A unitary 2-design is a $(k, \varepsilon)$-quantum spectral extractor with output size $m=(n+k) / 2-\log (1 / \sqrt{\varepsilon})$, where $n$ denotes the input size. 
Note that $k$ can be negative for entangled input states, and that the corresponding classical result for families of twouniversal hash functions reads $m=k-\log \frac{1}{\varepsilon}$ (Proposition 6 ).

Proof: For any $X_{N}, Y_{N} \in \mathcal{P}\left(\mathcal{H}_{N}\right)$ we get

$$
\begin{aligned}
& \left\langle X_{N} \mid\left(\tau^{\dagger} \circ \tau\right)\left(Y_{N}\right)\right\rangle=\left\langle\tau\left(X_{N}\right) \mid \tau\left(Y_{N}\right)\right\rangle \\
& =\operatorname{tr}\left[\operatorname{tr}\left[X_{N}^{\dagger}\right] \cdot \frac{\mathbb{1}_{M D}}{|M| \cdot|D|} \cdot \operatorname{tr}\left[Y_{N}\right] \cdot \frac{\mathbb{1}_{M D}}{|M| \cdot|D|}\right] \\
& =\frac{1}{|M| \cdot|D|} \cdot \operatorname{tr}\left[X_{N}^{\dagger}\right] \cdot \operatorname{tr}\left[Y_{N}\right] .
\end{aligned}
$$

Furthermore, we calculate

$$
\begin{gathered}
\left\langle X_{N} \mid\left(\psi^{\dagger} \circ \psi\right)\left(Y_{N}\right)\right\rangle=\frac{1}{|D|^{2}} \cdot \sum_{i=1}^{|D|}\left\langle X_{N} \mid\left(\psi_{i}^{\dagger} \circ \psi_{i}\right)\left(Y_{N}\right)\right\rangle \\
=\frac{1}{|D|^{2}} \cdot \sum_{i=1}^{|D|} \operatorname{tr}\left[X_{N}^{\dagger}\left(U_{N}^{i}\right)^{\dagger}\right. \\
\left.\left(\mathbb{1}_{N \backslash M} \otimes\left(\operatorname{tr}_{N \backslash M}\left[U_{N}^{i} Y_{N}\left(U_{N}^{i}\right)^{\dagger}\right]\right)\right) U_{N}^{i}\right] \\
=\frac{1}{|D|^{2}} \cdot \sum_{i=1}^{|D|} \operatorname{tr}\left[\left(\operatorname{tr}_{N \backslash M}\left[U_{N}^{i} X_{N}^{\dagger}\left(U_{N}^{i}\right)^{\dagger}\right]\right.\right. \\
\left.\left.\otimes \operatorname{tr}_{N^{\prime} \backslash M^{\prime}}\left[U_{N^{\prime}}^{i} Y_{N^{\prime}}\left(U_{N^{\prime}}^{i}\right)^{\dagger}\right]\right) F_{M M^{\prime}}\right] \\
=\frac{1}{|D|} \cdot \operatorname{tr}\left[\left(X_{N}^{\dagger} \otimes Y_{N^{\prime}}\right) \frac{1}{|D|} \sum_{i=1}^{|D|}\left(\left(U_{N}^{i}\right)^{\dagger} \otimes\left(U_{N^{\prime}}^{i}\right)^{\dagger}\right)\right. \\
\left.\left(F_{M M^{\prime}} \otimes \mathbb{1}_{N N^{\prime} \backslash M M^{\prime}}\right)\left(U_{N}^{i} \otimes U_{N^{\prime}}^{i}\right)\right]
\end{gathered}
$$

where we have used that the partial trace commutes with the identity, and denote the swap operator by $F_{M M^{\prime}}$. Since $\left\{U_{N}^{1}, \ldots, U_{N}^{|D|}\right\}$ is a unitary 2-design we have that [10, Lemma 3.4]

$$
\begin{aligned}
& \frac{1}{|D|} \sum_{i=1}^{|D|}\left(\left(U_{N}^{i}\right)^{\dagger} \otimes\left(U_{N^{\prime}}^{i}\right)^{\dagger}\right)\left(F_{M M^{\prime}} \otimes \mathbb{1}_{N N^{\prime} \backslash M M^{\prime}}\right)\left(U_{N}^{i} \otimes U_{N^{\prime}}^{i}\right) \\
& =\int\left(U_{N}^{\dagger} \otimes U_{N^{\prime}}^{\dagger}\right)\left(F_{M M^{\prime}} \otimes \mathbb{1}_{N N^{\prime} \backslash M M^{\prime}}\right)\left(U_{N} \otimes U_{N^{\prime}}\right) d U \\
& =\frac{1}{|M|} \cdot \frac{|N|^{3}-|M|^{2} \cdot|N|}{|N|^{3}-|N|} \cdot \mathbb{1}_{N N^{\prime}} \\
& \quad+\frac{1}{|M|} \cdot \frac{|N|^{2} \cdot|M|^{2}-|N|^{2}}{|N|^{3}-|N|} \cdot F_{N N^{\prime}}
\end{aligned}
$$

Hence, we arrive at

$$
\left\langle X_{N} \mid\left(\psi^{\dagger} \circ \psi-\tau^{\dagger} \circ \tau\right)\left(X_{N}\right)\right\rangle \leq \frac{|M|}{|N| \cdot|D|} \cdot\left\langle X_{N} \mid X_{N}\right\rangle,
$$

and the claim follows.

As in the classical case, quantum spectral extractors always have a long seed.

Proposition 14. Every $(k, \varepsilon)$-quantum spectral extractor with input size $n$, output size $m$, and seed size $d$ necessarily has $d \geq \min \{n-k, m\}+\log (1 / \varepsilon)-O(1)$.
Proof: Let $\mathcal{H}_{S} \subset \mathcal{H}_{M}$ with $|S|=\left\lceil 2^{k} \cdot|M| /|N|\right\rceil$, let $\{|t\rangle\}_{t=1}^{|N| /|M|}$ be an orthonormal basis of $\mathcal{H}_{N \backslash M}$, and consider the state

$$
\gamma_{N}=\frac{|M|}{|S| \cdot|N|} \cdot \sum_{s \in S} \sum_{t=1}^{|N| /|M|}\left(U_{N}^{1}\right)^{\dagger}|s t\rangle\left\langle\left. s t\right|_{N} U_{N}^{1} .\right.
$$

Since $H_{2}(N)_{\gamma}=-\log \left\|\gamma_{N}\right\|_{2}^{2} \geq k$ we have by the same arguments as in the classical case (Proposition 7 ) that

$$
\begin{aligned}
& \lambda_{1}\left(\psi^{\dagger} \circ \psi-\tau^{\dagger} \circ \tau\right) \\
& \geq \frac{2^{k}}{|D|^{2}} \cdot \sum_{i=1}^{|D|}\left\|\operatorname{tr}_{N \backslash M}\left[U_{N}^{i} \gamma_{N}\left(U_{N}^{i}\right)^{\dagger}\right]-\frac{\mathbb{1}_{M}}{|M|}\right\|_{2}^{2} \\
& \geq \frac{2^{k}}{|D|^{2}} \cdot\left\|\operatorname{tr}_{N \backslash M}\left[U_{N}^{1} \gamma_{N}\left(U_{N}^{1}\right)^{\dagger}\right]-\frac{\mathbb{1}_{M}}{|M|}\right\|_{2}^{2} \\
& \geq \frac{2^{k}}{|D|^{2}} \cdot|S| \cdot\left(\frac{1}{|S|}-\frac{1}{|M|}\right)^{2} .
\end{aligned}
$$

The rest of the proof proceeds as in the classical case (Proposition 77, except that we use in the very end a general lower bound for the seed of quantum extractors (Proposition 15. instead of the corresponding bound for classical extractors $(77$.

We show in the next section that there exists a quantum extractor with seed size $O(\log (1 / \varepsilon))$ matching the simple $d \geq$ $\log (1 / \varepsilon)$ lower bound. In contrast, any classical extractor has to satisfy $d \geq \log (n-k)+2 \log (1 / \varepsilon)-O(1)$.

Proposition 15. Every $(k, \varepsilon)$-quantum min-entropy extractor with $k \leq n-1$ ( $n$ is the output size) necessarily has seed size $d \geq \log (1 / \varepsilon)$.

Proof: Let $\mathcal{H}_{S} \subset \mathcal{H}_{M}$ with $|S|=|M| / 2$, let $\{|t\rangle\}_{t=1}^{|N| /|M|}$ be an orthonormal basis of $\mathcal{H}_{N \backslash M}$, and consider the state

$$
\gamma_{N}=\frac{2}{|M|} \cdot \sum_{s \in S} \sum_{t=1}^{|N| /|M|}\left(U_{N}^{1}\right)^{\dagger}|s t\rangle\left\langle\left. s t\right|_{N} U_{N}^{1} .\right.
$$

Since $H_{\min }(N)_{\sigma}=n-1$, and

$$
\begin{aligned}
& \left\|\operatorname{tr}_{N \backslash M}\left[U_{N}^{1} \gamma_{N}\left(U_{N}^{1}\right)^{\dagger}\right]-\frac{\mathbb{1}_{M}}{|M|}\right\|_{1} \\
& =\| \frac{2}{|M|} \cdot \sum_{s \in S}|s\rangle\left\langle\left. s\right|_{M}-\frac{\mathbb{1}_{M}}{|M|} \|_{1}\right. \\
& =1,
\end{aligned}
$$

the claim follows.

\section{Short Seeded Quantum Extractors}

Here we show that very small sets of random unitaries yield good quantum extractors.

Theorem 16. There exists a $(k, \varepsilon)$-quantum extractor with $m=(n+k) / 2-\log (1 / \varepsilon)-O(1)$, and $d=2 \log (1 / \varepsilon)+$ $O(\log \log (1 / \varepsilon))$. 
Proof: For $\rho_{N} \in \mathcal{S}\left(\mathcal{H}_{N}\right)$ with $H_{\min }(N)_{\rho} \geq l$ we have by the extraction property of unitary 2-designs (Proposition 13) that

$$
\int\left\|\operatorname{tr}_{N \backslash M}\left[U_{N} \rho_{N} U_{N}^{\dagger}\right]-\frac{\mathbb{1}_{M}}{|M|}\right\|_{1} d U \leq \frac{M}{\sqrt{N \cdot 2^{l}}},
$$

where the integration is with respect to the Haar measure on the unitary group. This means that for each specific input there exists a unitary $U_{N}$ that extracts well. We use a measure concentration argument (based on Lévy's lemma) that gives [9. Theorem 3.9],

$$
\begin{gathered}
\operatorname{Pr}\left\{\left\|\operatorname{tr}_{N \backslash M}\left[U_{N} \rho_{N} U_{N}^{\dagger}\right]-\frac{\mathbb{1}_{M}}{|M|}\right\|_{1} \geq \frac{M}{\sqrt{N \cdot 2^{l}}}+\gamma\right\} \\
\leq \exp \left(-\frac{N \gamma^{2} \cdot 2^{l}}{16}\right),
\end{gathered}
$$

for $\gamma>0$. Moreover, we use a concentration of the average bound [12, Lemma A.2] to get

$$
\begin{aligned}
\operatorname{Pr}\left\{\frac{1}{t} \cdot \sum_{i=1}^{t} \| \operatorname{tr}_{N \backslash M}\left[U_{N}^{i} \rho_{N}\left(U_{N}^{i}\right)^{\dagger}\right]-\right. & \left.\frac{\mathbb{1}_{M}}{|M|} \|_{1}-\frac{M}{\sqrt{N \cdot 2^{l}}} \geq \gamma\right\} \\
& \leq \exp \left(-\frac{t N \gamma^{2} \cdot 2^{l}}{16}\right) .
\end{aligned}
$$

In order to obtain a set of unitaries that extracts well for all states, we use a net $N_{l, \delta}$ of states with $\left|N_{l, \delta}\right| \leq(5 / \delta)^{2 N \cdot 2^{l}}$ such that for every flat $l$-source $\rho_{N}$ (i.e., $\rho_{N}$ has $2^{l}$ non-zero eigenvalues equal to $2^{-l}$ ) there exists $\bar{\rho}_{N} \in N_{l, \delta}$ with $\| \rho_{N}-$ $\bar{\rho}_{N} \|_{1} \leq \delta$ for (sufficiently small) $\delta>0$ [16, Lemma II.4]. For a union bound over all $\rho_{N} \in N_{l, \delta}$, 45 then gives

$$
\begin{gathered}
\operatorname{Pr}\left\{\exists \rho_{N} \in N_{l, \delta}: \frac{1}{t} \cdot \sum_{i=1}^{t}\left\|\operatorname{tr}_{N \backslash M}\left[U_{N}^{i} \rho_{N}\left(U_{N}^{i}\right)^{\dagger}\right]-\frac{\mathbb{1}_{M}}{|M|}\right\|_{1}\right. \\
\left.-\frac{M}{\sqrt{N \cdot 2^{l}}} \geq \gamma\right\} \leq\left(\frac{5}{\delta}\right)^{2 N \cdot 2^{l}} \cdot \exp \left(-\frac{t N \gamma^{2} \cdot 2^{l}}{16}\right) .
\end{gathered}
$$

Now, we fix $\frac{M}{\sqrt{N \cdot 2^{k}}}=\varepsilon / 3$ giving us $m=(n+k) / 2-$ $\log (1 / \varepsilon)-\log (3)$. Furthermore, we choose $\gamma=\varepsilon / 3$ and $t=\left(C / \varepsilon^{2}\right) \cdot \log (1 / \delta)$ for some (sufficiently large) $C>0$. From (46) we then get that for all $\rho_{N} \in N_{k, \delta}$,

$$
\frac{1}{t} \cdot \sum_{i=1}^{t}\left\|\operatorname{tr}_{N \backslash M}\left[U_{N}^{i} \rho_{N}\left(U_{N}^{i}\right)^{\dagger}\right]-\frac{\mathbb{1}_{M}}{|M|}\right\|_{1} \leq 2 \varepsilon / 3,
$$

with very high probability. By taking a union bound over all $l \geq k$, we get that 477 still holds with very high probability for all $\rho_{N} \in \bigcup_{l \geq k} N_{l, \delta}$. Hence we have shown the existence of a set of unitaries with $d=\log (t)=2 \log (1 / \varepsilon)+\log \log (1 / \delta)+$ $\log (C)$ that extracts well for all $\rho_{N} \in \bigcup_{l \geq k} N_{l, \delta}$. In order to make it work for all $\rho_{N} \in \mathcal{S}\left(\mathcal{H}_{N}\right)$ with $H_{\min }(N)_{\rho} \geq k$, we write $\rho_{N}$ as a mixture $\rho_{N}=\sum_{j} p_{j} \rho_{N}^{j}$ of flat $k$-sources $\rho_{N}^{j}$ [29, Lemma 6.10]. For each $\rho_{N}^{j}$, we know there exists $\bar{\rho}_{N}^{j} \in \cup_{l \geq k} N_{l, \delta}$ such that $\left\|\rho_{N}^{j}-\bar{\rho}_{N}^{j}\right\|_{1} \leq \delta$. This means that for all $\rho_{N}^{j}$ we have

$$
\frac{1}{t} \cdot \sum_{i=1}^{t}\left\|\operatorname{tr}_{N \backslash M}\left[U_{N}^{i} \rho_{N}^{j}\left(U_{N}^{i}\right)^{\dagger}\right]-\frac{\mathbb{1}_{M}}{|M|}\right\|_{1} \leq 2 \varepsilon / 3+\delta .
$$

The claim follows for $\delta=\varepsilon / 3$.

Thus the optimal seed size does only depend on the error $\varepsilon$ and not on the input size $n$ and min-entropy $k$ as in the classical case. However, we do not know if the extractor from Theorem 16 is also decoupling. Or more generally, if any decoupling quantum extractors with seed size $d<$ $\min \{n-k, m\}+\log (1 / \varepsilon)-O(1)$ exist (cf. Proposition 14).

\section{DISCUSSION}

We note that our stability result for classical and quantum spectral extractors (Theorems 4 and 11) also works if the quantum side information is described by infinite-dimensional Hilbert spaces.

There are many open questions whose answers would have applications in quantum information theory. Concerning classical extractors we would like to gain a general understanding of when a construction is quantum-proof. Following Ta-Shma [26], we mention that the example from [13] is compatible with the conjecture that every extractor is approximately quantum-proof with $\varepsilon \mapsto \varepsilon^{\prime}=O(\varepsilon \cdot m)$. For quantum extractors we would like to find probabilistic and explicit constructions that are decoupling but not based on spectral extractors. For quantum spectral extractors we would like to find probabilistic and explicit constructions that match our lower bound for the seed size in Proposition 14. Finally, quantum spectral extractors $\psi$ are specified by the second largest eigenvalue $\lambda_{2}\left(\psi^{\dagger} \circ \psi\right)$, and this relates them to the study of balanced quantum expanders (as, e.g., defined in [3]).

\section{ACKNOWLEDGMENT}

We acknowledge discussions with Stephanie Wehner. MB and VBS acknowledge financial support by the German Science Foundation (grant CH 843/2-1), the Swiss National Science Foundation (grants PP00P2-128455, 20CH21-138799 (CHIST-ERA project CQC)), the Swiss National Center of Competence in Research 'Quantum Science and Technology (QSIT)', the Swiss State Secretariat for Education and Research supporting COST action MP1006 and the European Research Council under the European Union's Seventh Framework Programme (FP/2007-2013) / ERC Grant Agreement no. 337603. VBS is in addition supported by an ETH Postdoctoral Fellowship. The research of OF is supported by the European Research Council grant No. 258932. OS acknowledges financial support by the Elite Network of Bavaria project QCCC

\section{REFERENCES}

[1] A. Ambainis and A. Smith. Small pseudo-random families of matrices: Derandomizing approximate quantum encryption. In Approximation, Randomization, and Combinatorial Optimization. Algorithms and Techniques, volume 3122 of LNCS, pages 249-260. 2004.

[2] G. Aubrun. On almost randomizing channels with a short Kraus decomposition. Communications in Mathematical Physics, 288:11031116, 2009. 
[3] A. Ben-Aroya, O. Schwartz, and A. Ta-Shma. Quantum expanders: Motivation and construction. Theory of Computing, 6(3):47-79, 2010.

[4] M. Berta, P. Coles, and S. Wehner. An equality between entanglement and uncertainty. 2013.

[5] M. Berta, J. M. Renes, and M. M. Wilde. Identifying the information gain of a quantum measurement. 2013.

[6] C. Dankert, R. Cleve, J. Emerson, and E. Livine. Exact and approximate unitary 2-designs and their application to fidelity estimation. Phys. Rev. A, 80:012304, Jul 2009.

[7] A. De, C. Portmann, T. Vidick, and R. Renner. Trevisan's extractor in the presence of quantum side information. SIAM Journal on Computing, 41(4):915-940, 2012.

[8] P. A. Dickinson and A. Nayak. Approximate randomization of quantum states with fewer bits of key. In AIP Conference Proceedings, volume 864, pages 18-36, 2006.

[9] F. Dupuis. The Decoupling Approach to Quantum Information Theory. $\mathrm{PhD}$ thesis, Université de Montréal, 2009.

[10] F. Dupuis, M. Berta, J. Wullschleger, and R. Renner. One-shot decoupling. 2010.

[11] F. Dupuis, O. Szehr, and M. Tomamichel. A decoupling approach to classical data transmission over quantum channels. 2012.

[12] O. Fawzi, P. Hayden, and P. Sen. From low-distortion norm embeddings to explicit uncertainty relations and efficient information locking. In Proceedings of the 43rd annual ACM symposium on Theory of computing, STOC '11, pages 773-782. ACM, 2011.

[13] D. Gavinsky, J. Kempe, I. Kerenidis, R. Raz, and R. de Wolf. Exponential separations for one-way quantum communication complexity, with applications to cryptography. In Proceedings of the thirty-ninth annual ACM symposium on Theory of computing, STOC '07, pages 516-525. ACM, 2007.

[14] D. Gross, K. Audenaert, and J. Eisert. Evenly distributed unitaries: On the structure of unitary designs. Journal of Mathematical Physics, 48(5):052104, 2007.

[15] A. Harrow and R. Low. Efficient quantum tensor product expanders and k-designs. In Approximation, Randomization, and Combinatorial Optimization. Algorithms and Techniques, volume 5687 of LNCS, pages 548-561. 2009.

[16] P. Hayden, D. Leung, P. W. Shor, and A. Winter. Randomizing quantum states: Constructions and applications. Communications in Mathematical Physics, 250:371-391, 2004.

[17] R. Konig and R. Renner. Sampling of min-entropy relative to quantum knowledge. Information Theory, IEEE Transactions on, 57(7):4760 4787, 2011

[18] R. Konig, R. Renner, and C. Schaffner. The operational meaning of minand max-entropy. Information Theory, IEEE Transactions on, 55(9):4337 -4347 , sept. 2009.

[19] R. Konig and B. Terhal. The bounded-storage model in the presence of a quantum adversary. Information Theory, IEEE Transactions on, 54(2):749-762, 2008.

[20] N. Nisan and D. Zuckerman. Randomness is linear in space. Journal of Computer and System Sciences, 51(1):43 - 52, 1996.

[21] R. Olkiewicz and B. Zegarlinski. Hypercontractivity in noncommutative Lp spaces. Journal of Functional Analysis, 161(1):246 - 285, 1999.

[22] J. Radhakrishnan and A. Ta-Shma. Bounds for dispersers, extractors, and depth-two superconcentrators. SIAM Journal on Discrete Mathematics, 13(1):2-24, 2000.

[23] R. Renner. Security of Quantum Key Distribution. PhD thesis, ETH Zurich, 2005.

[24] M. Sipser. Expanders, randomness, or time versus space. Journal of Computer and System Sciences, 36(3):379 - 383, 1988.

[25] O. Szehr. Decoupling theorems. Master's thesis, ETH Zurich, 2011.

[26] A. Ta-Shma. Extractors against classical and quantum adversaries. Tutorial QCrypt, 2013.

[27] M. Tomamichel, C. Schaffner, A. Smith, and R. Renner. Leftover hashing against quantum side information. Information Theory, IEEE Transactions on, 57(8):5524 -5535, aug. 2011.

[28] L. Trevisan. Construction of extractors using pseudo-random generators (extended abstract). In Proceedings of the thirty-first annual ACM symposium on Theory of computing, STOC '99, pages 141-148. ACM, 1999.

[29] S. P. Vadhan. Pseudorandomness. Lecture notes, 2011. 